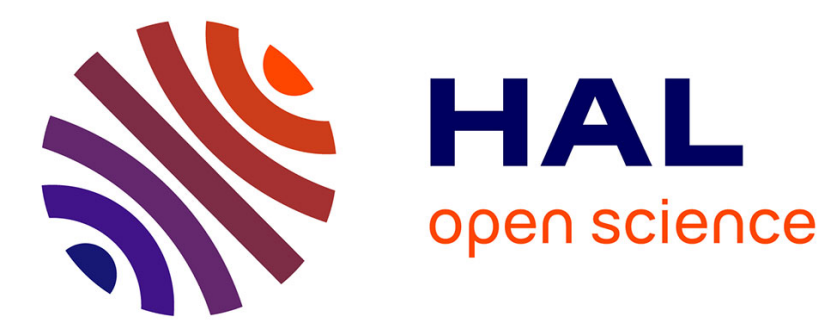

\title{
Finite element modelling of rate-dependent ratcheting in granular materials
}

\author{
Ali Karrech, Abdennour Seibi, Denis Duhamel
}

\section{To cite this version:}

Ali Karrech, Abdennour Seibi, Denis Duhamel. Finite element modelling of rate-dependent ratcheting in granular materials. Computers and Geotechnics, 2011, 38, pp.105-112. 10.1016/j.compgeo.2010.08.012 . hal-00668326

\section{HAL Id: hal-00668326 \\ https://hal.science/hal-00668326}

Submitted on 9 Feb 2012

HAL is a multi-disciplinary open access archive for the deposit and dissemination of scientific research documents, whether they are published or not. The documents may come from teaching and research institutions in France or abroad, or from public or private research centers.
L'archive ouverte pluridisciplinaire HAL, est destinée au dépôt et à la diffusion de documents scientifiques de niveau recherche, publiés ou non, émanant des établissements d'enseignement et de recherche français ou étrangers, des laboratoires publics ou privés. 


\title{
Finite Element Modeling of Rate-Dependent Ratcheting in Granular Materials
}

\author{
A. Karrech ${ }^{a, *}$, A. Seibi ${ }^{b}$, D. Duhamel ${ }^{c}$

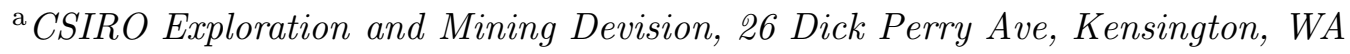 \\ 6151 Australia \\ ${ }^{\mathrm{b}}$ Mechanical Engineering Program, Petroleum Institute, PO Box 2533, Abu Dhabi, \\ $U A E$. \\ c Université Paris-Est, UR Navier, Ecole Nationale des Ponts et Chaussées, 6 et 8 \\ avenue Blaise Pascal, Cité Descartes, Champs sur Marne, 77455 \\ Marne-La-Vallée, Cedex 2, France
}

\begin{abstract}
The present paper introduces a comprehensive model capable of describing the behavior of granular materials under cyclic loading. Its main thrust is the introduction of the "Chicago" law in a continuum approach to account for the ratcheting effects. It also emphasizes the rate-dependency as a dissipative mechanism which acts independently or jointly with the ratcheting effect. Non-associated plasticity is adopted since the paper mainly targets the long term response of granular materials that are widely encountered in railway tracks and pavements. The numerical procedure is based on the return mapping algorithm where Newton's method is used to calculate the non-linear consistency parameter of the flow rule and obtain the consistent tangent modulus. The model was validated using numerical examples including multi-axial, and cyclic loading conditions.
\end{abstract}


Key words: Viscoplastic, Ratcheting, Granular materials, Mixed hardening, Numerical implementation.

\section{Introduction}

Granular materials are receiving increasing interest in modern industry due to their complex mechanical behavior which represents a challenging subject requiring a lot of research efforts. Different modeling approaches, based on discrete or continuum descriptions, have been used to study the response of these materials especially under cyclic loading. The discrete element methods such as the molecular dynamics (Cundall and Strack (1979, 1971); Gallas et al. (1992); Roux (2004); Karrech et al. (2007)) and contact dynamics (Azema et al. (2006); Saussine et al. (2005)) are well adapted to study samples consisting of finite number of particles. Although the discrete element methods describe accurately the local mechanisms of energy dissipation caused by ratcheting effects, loss of contacts, and granular flow, these techniques cannot treat samples made of large number of grains. Continuum approaches represent convenient time saving alternatives though limited in predicting local inter-granular mechanisms such as contact losses (Karrech et al. (2008)).

Research studies on cyclically loaded granular materials revealed that ratcheting, rate-dependency, and non associated flow are necessary features, which should be taken into account to formulate constitutive models (see Karrech (2007) and the references in there). Experimental studies of the same spirit showed that ratcheting can be described with a logarithmic function known as Chicago law (Ben-Naim et al. (1998); Nowak et al. (1998)). This empiri-

\footnotetext{
* Corresponding Author
}

Email address: kar10b@csiro.au (A. Karrech). 
cal law was confirmed numerically using discrete element methods by Rosato and Yacoub (2000), Philippe and Bideau (2001), and Rémond (2003). Both experimental and numerical results showed that granular materials exhibit a gradual decrease of the volume of voids resulting in a nonlinear permanent deformation with respect to the number of cycles. A similar decay was observed by Guérin et al. (1999) and Bodin et al. (2006) when studying the settlement of granular materials of railway platforms due to repeated loading. In addition, Saussine et al. (2005) developed a discrete element model based on the contact dynamics which showed the same trend. More recently, Karrech et al. (2007) developed a numerical algorithm based on molecular dynamics and showed that the logarithmic law is appropriate for long term settlement prediction in granular materials. The obtained empirical law proved to be an accurate description of the decaying phenomenon of cyclically loaded granular materials.

The present work is based on several existing computational procedures which involve the above mechanisms of energy dissipation within the framework of continuum mechanics. The purpose of this work is to combine some of the existing techniques and come-up with a comprehensive numerical tool which encompasses the observed logarithmic decay as well as the rate-dependency and frictional aspects. In this context Lu and Wright (1998) suggested a numerical model to describe the behavior of asphalt concrete under cyclic loading where an exponential empirical law was introduced to describe the permanent deformation. However, the cyclic effect was included in the rate-dependency mechanism rather than ratcheting. Lorefice et al. (2008) introduced a different model which was intended to describe the rate-dependent behavior of quasibrittle materials like concrete but without taking into account the ratcheting effect. Recently, Cvitanic et al. (2008) formulated a theoretical and numeri- 
cal framework which is based on the non-associated plasticity with isotropic hardening. The numerical integration included the return mapping technique which consists in projecting the trial stress on the surface of yielding at every increment (see also Key (1974); Dafalias (1977); Chen and Han (1987); Cernocky and Krempl (1979); Marques and Owen (1984); Loret and Prevost (1986)). This technique which was first introduced by Wilkins (1964) was improved by Simo and Taylor (1985) and Hofstetter et al. (1993) who proposed the consistent tangent modulus. Liu (2001); Kang (2004), Kumar and Nukala (2006) proved that the return mapping coupled with the consistent tangent modulus is an effective and robust tool to integrate nonlinear constitutive equations. Although, the cited models covered particular combinations of rate dependency, ratcheting, non-associated flows, and local compaction, none of them contains a comprehensive approach which takes into account these phenomena altogether to predict the response of granular materials.

The present study is developed in light of the above mentioned contributions while taking into account the (i) non associated flow rule, (ii) Drucker-Prager yield and plastic potential functions instead of the frequently used Von-Mises yield function, (iii) logarithmic ratcheting effect, and (iv) robust integration method based on the return mapping algorithm. This paper is divided into three major sections. The first section gives a brief description of the problem under consideration, defines the constitutive continuous model and highlights the assumptions adopted in the study. The second one describes the numerical procedure used to integrate the constitutive model. The last section is dedicated to the application of the developed model on particular examples. 


\section{Constitutive continuous model}

The constitutive model developed in this paper is based on a linear combination of kinematic and isotropic hardening where the strain increment is defined by:

$$
d \epsilon_{i j}=d \epsilon_{i j}^{e}+d \epsilon_{i j}^{i}+d \epsilon_{i j}^{\kappa}=d \epsilon_{i j}^{e}+d \epsilon_{i j}^{v p}
$$

The superscripts $e$ and $v p$ denote respectively the elastic and viscoplastic strains. The viscoplastic strain is decomposed into an isotropic strain, $d \epsilon_{i j}^{i}=$ $M d \epsilon_{i j}^{v p}$, and a kinematic strain, $d \epsilon_{i j}^{\kappa}=(1-M) d \epsilon_{i j}^{v p}$ where the constant $M$, describes the combination of both hardening effects. It takes the value 1 when the hardening is fully isotropic and 0 when it is fully kinematic. The elastic behavior is described with a linear law given by:

$$
d \sigma_{i j}=C_{i j k l}\left(d \epsilon_{k l}-d \epsilon_{k l}^{v p}\right)
$$

where $C_{i j k l}$ is a fourth order tensor describing the elastic behavior, $\epsilon_{i j}$ and $\sigma_{i j}$ are second order tensors denoting respectively the strain and Cauchy stress. The limit of elasticity is described by a Drucker-Prager yield function written as:

$$
f\left(\hat{\sigma}_{i j}\right)=f\left(\sigma_{i j}-\alpha_{i j}\right)=a_{1} \hat{I}_{1}+\sqrt{3 \hat{J}_{2}}=q
$$

where $\mathrm{f}$ is a convex function. The first and second invariants are expressed by $\hat{J}_{2}=\frac{1}{2} \hat{s}_{i j} \hat{s}_{i j}$ and $\hat{I}_{1}=\sigma_{i i}-\alpha_{i i}$, respectively. The terms $s_{i j}=\sigma_{i j}-p \delta_{i j}$,

$\hat{s}_{i j}=s_{i j}-\alpha_{i j}+\frac{1}{3} \alpha_{i i} \delta_{i j}, \mathrm{p}$, and $\delta_{i j}$ represent respectively the deviatoric stress, kinematic stress, hydrostatic pressure, and Kronecker identity. In this model, we express the translation or backstress $\alpha_{i j}$ using Prager's hardening rule as follows:

$$
d \alpha_{i j}=c d \epsilon_{i j}^{\kappa}
$$


In order to describe the ratcheting effect we express the Prager parameter $c$ in the above equation in terms of the number of loading cycles $N$. For a given periodic loading of frequency $\omega$, a cycle corresponds to a period of time $2 \pi \omega$. The Chicago logarithmic law obtained by Ben-Naim et al. (1998); Nowak et al. (1998); Rosato and Yacoub (2000); Philippe and Bideau (2001) and Rémond (2003) is used to express the kinematic hardening as follows:

$$
c(N)=\frac{c_{1} A}{1+c_{2} \ln \left(\frac{N}{\tau}+1\right)}
$$

where $c_{1}, c_{2}$, and $\tau$ are material constants that can be estimated experimentally. This logarithmic effect is mainly due to the compaction of granular materials subjected to repeated loading as explained by Guérin et al. (1999); Bodin et al. (2006); Saussine et al. (2005), and Karrech et al. (2007).

The term $q$ in Equation (3) can be expressed in terms of yield strength using the particular case of uniaxial loading as follows:

$$
f\left(\hat{\sigma}_{e}\right)=a_{1} \hat{\sigma}_{e}+\hat{\sigma}_{e}=q
$$

Therefore, it can be deduced that:

$$
\hat{\sigma}_{e}=\frac{a_{1} \hat{I}_{1}+\sqrt{3 \hat{J}_{2}}}{1+a_{1}}
$$

Furthermore, a plastic potential in accordance with the Drucker-Prager equation is considered:

$$
g\left(\hat{\sigma}_{i j}\right)=a_{2} \hat{I}_{1}+\sqrt{3 \hat{J}_{2}}
$$

If the constants $a_{1}$ and $a_{2}$ are different, the material is non-associated. The flow rule in this case is defined by:

$$
\dot{\epsilon}_{i j}^{v p}=\dot{\lambda} \frac{\partial g}{\partial \sigma_{i j}}
$$

where $\dot{\lambda}$ is a positive parameter which can be evaluated by selecting the ap- 
propriate viscous potential. In the present study, Perzyna's model is adopted to describe the rate-dependency. Therefore, the consistency parameter can be expressed as follows:

$$
\dot{\lambda}=\frac{\psi(f)}{\zeta}
$$

where $\zeta$ is a fluidity parameter and $\psi$ is a monotonically increasing function defined by: $\psi(f)=f$ if $f \geq 0$ and $\psi(f)=0$ otherwise. By defining $\bar{f}=$ $f-\psi^{-1}(\dot{\lambda} \zeta)$ and using the principle of maximum dissipation, it can be seen that the Kuhn-Tucker conditions hold during the viscoplastic flow:

$$
\dot{\lambda} \bar{f}=0, \quad \dot{\lambda} \geq 0, \text { and } \bar{f} \leq 0
$$

These conditions can be used to determine the factor $\dot{\lambda}$ as will be shown in the next sections. Note that unlike the model of Suiker and Borst (2003) where the plastic strain evolution is expressed in terms of number of cycles, rate dependency in our approach requires an explicit dependency of the inelastic strain on time. This means that ratcheting and rate dependency can act as independent mechanisms.

\section{Finite element Implementation}

The algorithm used to integrate the developed non-linear material behavior is similar to those adopted for rate-independent cases. A computational procedure based on the implicit return mapping scheme developed by Simo and Taylor (1985) for associated plasticity and extended by Cvitanic et al. (2008) for non-associated plasticity, is adopted in the present work to take into account the rate dependency of granular materials. A detailed description of the algorithm developed in this study is presented. 


\subsection{Return mapping}

The return mapping starts from a converged configuration, $\mathcal{C}_{n}\left(\boldsymbol{\sigma}_{n}, \boldsymbol{\epsilon}_{n}^{v p}\right)$, where the state variables are known to deduce the new configuration, $\mathcal{C}_{n+1}\left(\boldsymbol{\sigma}_{n+1}, \boldsymbol{\epsilon}_{n+1}^{v p}\right)$, which satisfies the material behavior. There are various techniques which can be used to integrate the behavior of materials (Simo and Taylor (1985); Hofstetter et al. (1993)). Most of them are based on predictor-corrector schemes which generally involve two steps. The first step uses the converged configuration, $\mathcal{C}_{n}$, Hooke's law (2), and a deformation increment, $\Delta \epsilon$, which can be evaluated using the incremental deformation theory (Cvitanic et al. (2008)) in order to calculate a trial elastic stress tensor as follows:

$$
\sigma_{i j}^{T}=\left(\sigma_{i j}\right)_{n}+C_{i j k l} \Delta \epsilon_{k l}^{e}
$$

If the elastic trial state does not violate the limit of elasticity, then it represents the solution of the problem. However, if the trial state is not admissible, a correction is required. The trial stress represents a starting point of the viscoplastic correction step which leads to the following result:

$$
\left(\sigma_{i j}\right)_{n+1}=\left(\sigma_{i j}\right)_{n}+C_{i j k l}\left(\Delta \epsilon_{k l}-\Delta \epsilon_{k l}^{v p}\right)=\sigma_{i j}^{T}-C_{i j k l} \Delta \epsilon_{k l}^{v p}
$$

The term on the right hand side of (13) is the correction or trial stress. The viscoplastic strain increment can be obtained from Equation (9) as follows:

$$
\Delta \epsilon_{i j}^{v p}=\Delta \lambda \frac{\partial g}{\partial \sigma_{i j}}
$$

\subsection{Consistency factor}

One of the main features of the computational procedure is the computation of the consistency factor, which allows the projection of the trial state on the surface $\bar{f}=0$, for correction. Since the evaluation of the stress-strain 
relationship takes a considerable amount of computing time, the efficiency of the algorithm used to integrate the material behavior is crucial. In particular, the algorithm suggested herein uses Newton's method to calculate the consistency parameter and deduce the equivalent viscoplastic strain which is evaluated only once. Jointly with the consistent tangent modulus, which will be developed in the next subsection, this technique guarantees a high rate of convergence. The procedure is based on the enforcement of the consistency condition, which can be symbolically written in a scalar form as follows:

$$
\Gamma(\Delta \lambda)=\bar{f}\left(\left(\hat{\sigma}_{i j}\right)_{n+1}\right)=0
$$

using Equations (3), (7), and (13), it can be seen that

$$
\Gamma(\Delta \lambda)=\bar{f}\left(\hat{\sigma}_{i j}^{T}-C_{i j k l} \Delta \epsilon_{k l}^{v p}-c(1-M) \Delta \epsilon_{k l}^{v p}\right)=0
$$

Equation (16) is approximated to the first order using Taylor Seris as follows:

$$
\Gamma(\Delta \lambda)=\bar{f}\left(\hat{\sigma}_{i j}^{T}\right)-\Delta \lambda \frac{\partial \bar{f}}{\partial \sigma_{i j}} C_{i j k l} \frac{\partial g}{\partial \sigma_{k l}}-\Delta \lambda c(1-M) \frac{\partial \bar{f}}{\partial \sigma_{i j}} \frac{\partial g}{\partial \sigma_{i j}}=0
$$

Assume that after a given iteration of the Newton's method, an approximation $\Delta \lambda$ to the consistency factor is obtained. Let $\delta(\Delta \lambda)$ be the difference between this value and the exact solution to the consistency condition. This implies that:

$$
\Gamma(\Delta \lambda+\delta(\Delta \lambda))=0
$$

Expanding the above equation in a Taylor series to the first order about the approximate consistency factor results in the following expression:

$$
\Gamma(\Delta \lambda)=-\delta(\Delta \lambda) \frac{\partial \Gamma(\Delta \lambda)}{\partial(\Delta \lambda)}
$$

Therefore, combining equations (17) and (19) leads to: 


$$
\delta(\Delta \lambda)=\frac{f\left(\sigma_{i j}^{n+1}\right)-q-\dot{\lambda} \zeta-\Delta \lambda \frac{\partial \bar{f}}{\partial \sigma_{k l}} C_{i j k l} \frac{\partial \bar{f}}{\partial \sigma_{k l}}-\Delta \lambda c(1-M) \frac{\partial \bar{f}}{\partial \sigma_{i j}} \frac{\partial g}{\partial \sigma_{i j}}}{\frac{\partial \Gamma(\Delta \lambda)}{\partial(\Delta \lambda)}}
$$

Equation (20) represents the correction of the consistency factor, which can be solved using Newton's method. The iterations consist in calculating $\delta(\Delta \lambda)$ and updating the consistency factor in an additive sense such that: $\Delta \lambda^{\text {new }}=$ $\Delta \lambda^{\text {old }}+\delta(\Delta \lambda)$ until convergence is achieved. Once the consistency factor is known the stresses and strains can be deduced.

\subsection{Consistent tangent modulus}

Differentiating the incremental equation (13) and using equation (14), results in:

$$
d \Delta\left(\sigma_{i j}\right)_{n+1}=C_{i j k l}\left(d \Delta \epsilon_{k l}-d \Delta \lambda \frac{\partial g}{\partial \sigma_{i j}}-\Delta \lambda \frac{\partial^{2} g}{\partial \sigma_{i j} \partial \sigma_{k l}} d \Delta\left(\sigma_{i j}\right)_{n+1}\right)
$$

Therefore, it can be deduced that:

$$
d \Delta\left(\sigma_{i j}\right)_{n+1}=D_{i j k l}\left(d \Delta \epsilon_{k l}-d \Delta \lambda \frac{\partial g}{\partial \sigma_{i j}}\right)
$$

where $D_{i j k l}=\left(C_{i j k l}^{-1}+\Delta \lambda \frac{\partial^{2} g}{\partial \sigma_{i j} \partial \sigma_{k l}}\right)^{-1}$. The consistency condition $\dot{\bar{f}}=0$ can be applied in order to deduce the consistent tangent modulus:

$$
d \Delta\left(\sigma_{i j}\right)_{n+1}=\left(D_{i j k l}-\frac{B_{i j} B_{k l}^{*}}{H^{*}}\right) d \Delta \epsilon_{k l}
$$

where $B_{k l}=D_{i j k l} \frac{\partial \bar{f}}{\partial \sigma_{i j}}, B_{k l}^{*}=D_{i j k l} \frac{\partial g}{\partial \sigma_{i j}}$, and $H^{*}=\frac{\partial \bar{f}}{\partial \sigma_{i j}} D_{i j k l} \frac{\partial g}{\partial \sigma_{i j}}+\left(3 a_{1} a_{2}+\right.$ $3 / 2)(1-M) c+\frac{M\left(1+a_{1}\right)^{2}\left(a_{2} \hat{I}_{1}+\sqrt{3 \hat{J}_{2}}\right) H_{p}}{q}+\frac{\zeta \dot{\lambda}}{\Delta t}$. In order to calculate $D_{i j k l}$, it is necessary to note that:

$$
\frac{\Delta \lambda \partial^{2} g}{\partial \sigma_{i j} \partial \sigma_{k l}}=\frac{3}{2} \frac{\Delta \lambda}{X}\left(\frac{\delta_{i k} \delta_{j l}+\delta_{i l} \delta_{j k}}{2}-\frac{\delta_{i j} \delta_{k l}}{3}-\frac{3}{2} \frac{\hat{s}_{i j} \hat{s}_{k l}}{X^{2}}\right)
$$


where $X=\sqrt{3 J_{2}}$. Hence, it can be deduced that:

$$
D_{i j k l}=\left(C_{i j k l}^{-1}+\frac{3}{2} \frac{\Delta \lambda}{X}\left(\frac{\delta_{i k} \delta_{j l}+\delta_{i l} \delta_{j k}}{2}-\frac{\delta_{i j} \delta_{k l}}{3}-\frac{3}{2} \frac{\hat{s}_{i j} \hat{s}_{k l}}{X^{2}}\right)\right)^{-1}
$$

It can be noticed that the above tensor can be written as follows:

$$
D_{i j k l}=\left(E_{i j k l}^{-1}-\frac{\Delta \lambda}{X} v_{i j} v_{k l}\right)^{-1}
$$

After some simple mathematical identification it can be seen that:

$$
E_{i j k l}=\gamma\left(K-\frac{2}{3} G\right) \delta_{i j} \delta_{k l}-\frac{3 G \Delta \lambda}{X} \gamma K \delta_{i j} \delta_{k l}+2 \gamma G \frac{\delta_{i k} \delta_{j l}+\delta_{i l} \delta_{j k}}{2}
$$

where $\gamma=\frac{X}{X+3 G \Delta \lambda}$. Since $\frac{\Delta \lambda}{X}<<1$, therefore, a first order development results in $\gamma \approx 1-\frac{3 G \Delta \lambda}{X}$ which means that $\frac{3 G \Delta \lambda}{X} \gamma \approx \frac{3 G \Delta \lambda}{X}$. Therefore,

$$
E_{i j k l}=\left(K-\frac{2}{3} \gamma G\right) \delta_{i j} \delta_{k l}+2 \gamma G \frac{\delta_{i k} \delta_{j l}+\delta_{i l} \delta_{j k}}{2}
$$

When $\frac{\Delta \lambda}{X}<<1$ approaches zero, $\gamma$ tends to 1 . Therefore, it can be seen that the inverse of $E_{i j k l}$ can be reduced to $C_{i j k l}$, in the above mentioned particular case. Applying Sherman-Morisson formula, Equation (26) can be rewritten as:

$$
D_{i j k l}=E_{i j k l}-\frac{v_{i j} E_{i j k l} E_{i j k l} v_{k l}}{v_{i j} E_{i j k l} v_{k l}-\frac{X}{\Delta \lambda}}
$$

where $v_{i j}=\frac{3}{2} \frac{\hat{s}_{i j}}{X}$. Substituting $v_{i j}$ and $E_{i j k l}$ in Equation (29) leads to the following expression.

$$
D_{i j k l}=\left(K-\frac{2}{3} \gamma G\right) \delta_{i j} \delta_{k l}+\gamma 2 G \frac{\delta_{i k} \delta_{j l}+\delta_{i l} \delta_{j k}}{2}+\beta \frac{\hat{s}_{i j}}{X} \frac{\hat{s}_{k l}}{X}
$$

where $\beta=-\frac{(3 \gamma G)^{2}}{3 \gamma G-\frac{X}{\Delta \lambda}}$. Since $\frac{\Delta \lambda}{X}<<1$, then $\beta \approx-\frac{(3 \gamma G)^{2} \Delta \lambda}{X}$. Using Equation (30), the terms of Equation (23) representing the consistent operator can be deduced:

$$
\begin{aligned}
& B_{i j}=D_{i j k l} \frac{\partial \bar{f}}{\partial \sigma_{k l}}=3 K a_{1} \delta_{i j}+(3 \gamma G+\beta) \frac{\hat{s}_{i j}}{X} \\
& B_{k l}^{*}=D_{i j k l} \frac{\partial g}{\partial \sigma_{i j}}=3 K a_{2} \delta_{k l}+(3 \gamma G+\beta) \frac{\hat{s}_{k l}}{X}
\end{aligned}
$$




$$
\frac{\partial \bar{f}}{\partial \sigma_{i j}} D_{i j k l} \frac{\partial g}{\partial \sigma_{k l}}=9 \beta K a_{1} a_{2}+(3 \gamma G+\beta)
$$

Therefore

$$
\begin{aligned}
H *= & 9 \beta K a_{1} a_{2}+(3 \gamma G+\beta)+\left(3 a_{1} a_{2}+3 / 2\right)(1-M) c \\
& +\frac{M\left(1+a_{1}\right)^{2}\left(a_{2} \hat{I}_{1}+\sqrt{3 \hat{J}_{2}}\right) H_{p}}{q}+\frac{\zeta \dot{\lambda}}{\Delta t}
\end{aligned}
$$

It is worth noticing that this work presents a general formulation of the problem which can be reduced to known particular cases: (i) If $\alpha_{1}=\alpha_{2}=0$, the yield and potential functions reduce from Drucker-Prager's to Von-Mises's form which is suitable for metallic materials. Most of the existing formulations where rate-dependency and mixed hardening are implemented can be classified within this category. For instance, Mayama et al. (2007) developed a constitutive model for cyclically loaded viscoplastic stainless steel at room temperature. Kanga et al. (2003) suggested a similar model where isotropic and kinematic hardening effects were considered and validated the model through an experimental investigation on stainless steel at ambient temperature. Kumar and Nukala (2006) introduced a constitutive model which is based on multi-component forms of kinematic and isotropic hardening variables in conjunction with Von Mises yield criterion. (ii) If the constant M equals 1 then our hardening description reduces to the isotropic case where material ratcheting does not play a tangible role. The formulation then reduces to the elastoviscoplastic model of Cela (1998) where inviscid description follows DruckerPrager's equation. This description was used to predict the response of reinforced concrete structures subjected to dynamic loads (see also Cvitanic et al. (2008) for the integration of an elasto-plastic behavior without ratcheting). (iii) When $\zeta$ reduces to zero then the model produces the ratcheting effects in rate-independent materials (see for instance Chen and Han (1987)). 


\section{Numerical Examples}

The numerical procedure at hand was implemented into the general purpose finite element code ABAQUS using the user material subroutine UMAT. In this section, we first examine the validity of the model through simple examples and then apply it to a particular case which is relevant to granular materials subjected to repeated loading. The material parameters used for simulation are summarized as follows: Young Modulus: $E=30 M P a$, Poisson's ratio: $\nu=0.3$, fluidity coefficient: $\zeta=5 \mathrm{MPas}$, friction angle: $\varphi=35^{\circ}$, dilatation angle: $\psi=10^{\circ}$, mixing hardening constant: $M=0.5$, and Chicago law parameters: $c_{1}=20 M P a, c_{2}=2$, and $\tau=20$. Isotropic hardening was described by the following stress-strain input $\left(\epsilon^{v p}, \sigma_{e}(\mathrm{MPa})\right)=\{(0 ., 0.2),(0.001$, $0.3),(0.005,0.34),(0.01,0.4),(0.05,0.6)\}$. These parameters can be identified experimentally using triaxial tests at different rates of loading. The constants $a_{1}$ and $a_{2}$ can be related to the friction and dilatation angles, $\varphi$ and $\psi$ respectively:

$$
a_{1}=-\frac{2 \sin (\varphi)}{3(3-\sin (\varphi))} \quad \text { and } \quad a_{2}=-\frac{2 \sin (\psi)}{3(3-\sin (\psi))}
$$

It can be noticed that the model is non associated since $a_{1}$ is different than $a_{2}$

\subsection{Cylindrical sample under monotonic triaxial loading}

This example consists of a cylindrical sample with unit radius and length (see Figure 1). Radial and axial displacements are applied on the upper and circumferential faces while the bottom side is constrained from moving in the axial direction. It is assumed that $\zeta=c=0$ in this particular case, in order to examine the capability of the model in predicting the frictional Drucker-Prager

behavior. We first calculate the analytical solution and then use the necessary 
simplifications to compare it with the numerical results. The displacement field which governs the motion takes the form:

$$
\boldsymbol{x}=\boldsymbol{\phi}(\boldsymbol{X}, t)=\boldsymbol{X}-a(t) Z \boldsymbol{e}_{z}-b(t) R \boldsymbol{e}_{r}
$$

Therefore, the total rate of stretches can be written as follows:

$$
\boldsymbol{d}=-\frac{\dot{a}}{1-a}\left(e_{r} \otimes e_{r}+e_{\theta} \otimes e_{\theta}\right)-\frac{\dot{b}}{1-b} \mathrm{e}_{z} \otimes e_{z}
$$

The rate of plastic stretches can be expressed as:

$$
\boldsymbol{d}^{p}=\dot{\lambda} \frac{\partial g}{\partial \boldsymbol{\sigma}}=\dot{\lambda}\left(\alpha_{2} \delta_{i j}+\frac{3}{2} \frac{s_{i j}}{X}\right)
$$

Hence, knowing that the mapping (36) creates no rotation, it can be deduced that the evolution of Cauchy stresses can be written as follows:

$$
\dot{\boldsymbol{\sigma}}=\left(K-\frac{2}{3} G\right) \operatorname{tr}\left(\boldsymbol{d}-\boldsymbol{d}^{p}\right) \mathbf{1}+2 G\left(\boldsymbol{d}-\boldsymbol{d}^{p}\right)
$$

If the response is purely elastic then $\boldsymbol{d}^{p}=\mathbf{0}$. After integration of Equation (39) it can be seen that the elastic solution within the hypothesis of small perturbations reads:

$$
\begin{aligned}
& \sigma_{z z}=-\left(K-\frac{2}{3} G\right)(2 a+b)-2 G b \\
& \sigma_{r r}=-\left(K-\frac{2}{3} G\right)(2 a+b)-2 G a \\
& \sigma_{\theta \theta}=\sigma_{r r}, \quad \sigma_{r \theta}=\sigma_{r z}=\sigma_{\theta z}=0
\end{aligned}
$$

Note that we used the first order development $\ln (1+x) \approx x$ for $x<<1$, since the displacements are small. The above equation can be used simultaneously with the first limit of elasticity $a_{1}\left(\sigma_{r r}+\sigma_{\theta \theta}+\sigma_{z z}\right) / 3+\sqrt{3 J_{2}}=q_{0}$ to evaluate the solution at the plasticity threshold.

The inelastic phase involves the plastic rate of displacements. Assuming that 
the small deformation theory holds and combining equations (38) and (37-39) results in the stress state:

$$
\begin{aligned}
& \sigma_{z z}=-\left(K-\frac{2}{3} G\right)(2 a+b)-2 G b+3 a_{2} \lambda K+3 \lambda G \frac{s_{z z}}{X} \\
& \sigma_{r r}=-\left(K-\frac{2}{3} G\right)(2 a+b)-2 G a+3 a_{2} \lambda K+3 \lambda G \frac{s_{r r}}{X} \\
& \sigma_{\theta \theta}=\sigma_{r r}, \quad \sigma_{r \theta}=\sigma_{r z}=\sigma_{\theta z}=0
\end{aligned}
$$

Hence, the solution of the problem can be presented in terms of pressure and deviatoric stress as follows:

$$
\begin{aligned}
& q_{d e v}=2 G(b-a)+3 \lambda G \\
& p=-K(2 a+b)-3 \lambda a_{2} K \\
& q\left(\epsilon_{p}\right)=-3 a_{1}(2 a+b) K-9 a_{1} a_{2} \lambda+2(b-a) G+3 \lambda G
\end{aligned}
$$

Figures 2 and 5 show a comparison between the analytical and numerical results. It can be seen that all figures show good agreement between both methods. This demonstrates the accuracy of the numerical approach developed in this study in predicting the response of an elasto-plastic material obeying to the yield and plastic functions of Drucker-Prager.

\subsection{Cylindrical sample under monotonic triaxial loading rates}

In this paragraph, we study the response of the same sample (see Figure 1) but we subject it to a confining pressure of $0.07 \mathrm{MPa}$ and a deviatoric stress of $0.275 \mathrm{MPa}$. We also use the same material properties and add a fluidity parameter $\zeta=5 M P$ as to take into account the rate dependency. Unfortunately, an analytical solution turned out to be difficult to obtain because of the additional non-linear viscous terms. 
Figure 6 shows the sample's response under different strain rates. The curves indicate that the material becomes stiffer when the loading rate increases. It is worth noting that the curve (a) in the figure represents the rate independent case which is identical to the results shown in figure 2 .

\subsection{Cylindrical sample under cyclic triaxial loading}

This example consists in subjecting a cylindrical sample to a confining pressure of $0.07 \mathrm{MPa}$ and a sinusoidal deviatoric loading of the form $S(t)=$ $S_{0}\left(1-\cos \left(\omega_{0} t\right)\right)$, where the loading amplitude is $S_{0}=0.09 \mathrm{MPa}$ and the circular frequency is $\omega_{0}=4 \pi s^{-1}$ (see Figure 11). The rate-dependent effect is not taken into account in this particular example. To demonstrate the accuracy of the model, different time increments were used. The shortest time increment produces the closer results to the exact solution. Figure 7 shows the strain response corresponding to the applied load which indicates a good accuracy for different time increments.

The analysis also treated the variation of the radial and axial displacements as well as the volumetric change with respect to the number of cycles as shown in Figures 8-10. Figures 8 and 9 show that the permanent displacements decrease with respect to the number of cycles. This result was also observed by Karrech (2007), using the molecular dynamics (discrete element method) to study the ratcheting effect in granular materials subjected to cyclic loading. On the other hand, Figure 10 shows the logarithmic compaction with respect to the number of cycles in accordance with the introduced Chicago law. 


\section{Application: partially confined viscoplastic sample under cyclic loading}

In this section, the developed model is now used to simulate the response of a partially confined cylindrical sample under cyclic loading. The sample has a radius $R=0.075 \mathrm{~m}$ and a length $L=0.15 \mathrm{~m}$. It is subjected to a cyclic loading of the form $S(t)=S_{0}\left(1-\cos \left(\omega_{0} t\right)\right)$ where circular frequency $\omega_{0}$ is varied. Figure 11 shows that the bottom surface of the sample is constrained from moving in the axial direction, $\underline{e}_{z}$, and the lateral surface is constrained from moving in the radial direction, $\underline{e}_{r}$. However, the top surface of the sample is partially loaded with an axial stress within the region of $r<0.5 R$. The material properties presented in the last section are used for simulation.

One of the important applications of the proposed model is the prediction of the accumulated permanent deformation in track platforms due to repeated loading. Figure 12 shows the variation of axial displacements at the central point $(\mathrm{r}=0, \mathrm{z}=\mathrm{L})$, with respect to the number of cycles for various loading frequencies. It can be seen that the axial displacement is decaying for all frequencies as the number of cycles increases. This leads to an increasing accumulation of permanent deformation. This result is in agreement with the

phenomena observed by Guérin et al. (1999) Bodin et al. (2006). The figure also shows an increase of the permanent deformation slope when the frequency increases from 1 to $100 \mathrm{~Hz}$. The accumulation of permanent deformation along the surface of the sample is plotted in figure 13 for a frequency of $10 \mathrm{~Hz}$ and different number of cycles. 


\section{Conclusion}

A comprehensive viscoplastic constitutive model describing the behavior of non-associated ratcheting granular materials is developed and implemented in finite element. One of the main features of the model is the incorporation of Chicago's law which describes the compaction of granular materials. The model was based on Drucker-Prager yield and potential functions and involves a rate dependency behavior described by Perzyna's theory. The integration of the model was performed by applying the well known predictor-corrector algorithm used for stress state estimation coupled with Newton's method for computing the consistency factor as well as the consistent tangent viscoplastic operator.

The benchmarking of the model was performed through specific examples. Good agreements between the analytical and computational results were obtained in case of a multi-axial loading of a frictional sample. This demonstrated the capability of the model in predicting one of relevant features of granular materials. The model was then used to describe the effect of ratcheting and rate dependency on a specific field case. The logarithmic permanent deformation which was observed experimentally in cyclically loaded materials was produced.

Future research work will take into account the dynamic aspects as recent experimental work proved that the frequency affects the density profiles in loaded samples. The transition from low to high loading frequencies is of particular interest, as the materials undergo phase's transformation from solid like to fluid like behavior due to vibration. 


\section{References}

Azema, E., Radjai, F., Peyroux, R., Dubois, F., and Saussine, G. (2006). Vibrational dynamics of confined granular material. Physical Review E, 74:031302.

Ben-Naim, E., Knight, J., and Nowak, E. (1998). Slow relaxation in granular compaction. Physica D, 123:380-384.

Bodin, V., Tamagny, P., Sab, K., and Gautier, P.-E. (2006). Détermination expérimentale d'une loi de tassement du ballast des voies férrées soumises à un chargement latéral. Canadian Geotechnical Journal, 43:pp. 1028-1041.

Cela, J. (1998). Analysis of reinforced concrete structures subjected to dynamic loads with a viscoplastic drucker-prager model. Applied Mathematical Modelling, 22:495-515.

Cernocky, E. P. and Krempl, E. (1979). A non-linear uniaxial integral constitutive equation incorporating rate effects creep and relaxation. International Journal Non-Linear Mechanics, 14:183-203.

Chen, W. and Han, D. (1987). Plasticity for Strctural Engineers. SpringerVerlag, New York.

Cundall, P. A. and Strack, O. D. L. (1971). A computer model for simulating progressive large scale movement of blocky rock systems. Proceedings of the Symposium of the International Society of Rock Mechanics, Nancy, France, $1(8)$.

Cundall, P. A. and Strack, O. D. L. (1979). A discrete numerical model for granular assemblies. Géotechnique, 29:47-65.

Cvitanic, V., Vlak, F., and Lozina, Z. (2008). A finite element formulation based on non-associated plasticity for sheet metal forming. International Journal of Plasticity, 24:646-687. 
Dafalias, Y. F. (1977). Elasto-plastic coupling within a thermodynamic strain space formulation of plasticity. International Journal Non-Linear Mechanics, $12: 327-337$.

Gallas, J., Herrmann, H., and Sokolowski, S. (1992). Convection cells in vibrating granular media. Physical Review Letters, 69(9):1371-1375.

Guérin, N., Sab, K., and Moucheront, P. (1999). Identification expérimentale d'une loi de tassement du ballast. Canadian Geotechnical Journal, 36:pp. $523-532$.

Hofstetter, G., Simo, J. C., and Taylor, R. L. (1993). A modified cap model: closest point solution algorithms. Computer and structures, 46(2):203-214.

Kanga, G., Ohnob, N., and Nebuc, A. (2003). Constitutive modeling of strain range dependent cyclic hardening. International Journal of Plasticity, 19:1801-1819.

Kang, G. (2004). A viscoplastic constitutive model for ratcheting of cyclically stable materials and its finite element implementation. Mechanics of Materials, 36:299-213.

Karrech, A., Duhamel, D., Bonnet, G., Roux, J. N., Chevoir, F., Canou, J., Dupla, J. C., and Sab, K. (2007). A computational procedure for the prediction of settlement in granular materials under cyclic loading. Computer Methods in Applied Mechanics and Engineering, 197(1-4):80-94.

Karrech, A., Duhamel, D., Bonnet, G., Roux, J. N., Chevoir, F., Canou, J., and Dupla, J. C. (2008). A causality analysis of settlement versus contacts loss in vibrated granular materials. Granular Matter, 8(1-4):195-204.

Karrech, A. (2007). Comportement des materiux granulaires sous vibration: Application au cas du ballast. PhD thesis, Ecole Nationale des Ponts et Chaussées, Paris, France.

Key, W. (1974). A finite element“" procedure for the large deformation dynamic 
response of axysimmetric solids. Computer Methods in applied mecchanis and engineering, 4:195-218.

Kumar, Q. P. and Nukala, V. (2006). A return mapping algorithm for cyclic viscoplastic constitutive models. Computer Methods in Applied Mechanics and Engineering, 195:148-178.

Liu, G. P. Y. (2001). Implicit consistent and continuum tangent operators in elastoplastic boundary element formulation. Computer Method in applied mechanics and engineering, 190:2157-2179.

Lorefice, R., Etse, G., and Carol, I. (2008). Viscoplastic approach for ratedependent failure analysis of concrete joints and interfaces. Accepted for publication in the International Journal of Solids and Structures.

Loret, B. and Prevost, J. (1986). Accurate numerical solutions for druckerprager elastic-plastic. Computer Method in applied mechanics and engineering, 54:259-277.

Lu, Y. and Wright, P. (1998). Numerical approach of visco-elastoplastic analysis for asphalt mixtures. Computers and Structures, 69:139-147.

Marques, J. M. M. C. and Owen, D. R. J. (1984). Some reflextions on elastopalstic stress calculation in finite element analysis. Computers and Structures, 18(6):1135-1139.

Mayama, T., Sasaki, K., and Ishikawa, H. (2007). A constitutive model of cyclic viscoplasticity considering changes in subsequent viscoplastic deformation due to the evolution of dislocation structures. International Journal of Plasticity, 23:915-930.

Nowak, E. R., Knight, J. B., Ben-Naim, E., Jaeger, H. M., and Nagel, S. R. (1998). Density fluctuations in vibrated granular materials. Physical Review E. Statistical Physics, 57(2):1971-1982.

Philippe, P. and Bideau, D. (2001). Numerical model for granular compaction 
under vertical tapping. Physical Review E., 63:0513041-05130410.

Rémond, S. (2003). Simulation of the compaction of confined mono-sized spherical particles systems under symmetric vibration. Physica A, 329:127146.

Rosato, A. D. and Yacoub, D. (2000). Microstructure evolution in compacted granular beds. Powder Technology, 109:255-261.

Roux, J. N. (2004). Elasticity, quasistatic deformation, and internal state of sphere packings. 17th ASCE Engineering Mechanics Conference, June 13-16 2004, Newark, DE.

Saussine, G., Cholet, C., Gautier, P., Dubois, F., Bohatier, C., and Moreau, J. (2005). Modelling ballast behavior under dynamic loading, part1: A 2d polygonal discrete element method approach. Computer Method in Applied Mechanics and Enginnering, 195(19-22):2841-2859.

Simo, J. C. and Taylor, R. L. (1985). Consistant tangent operators for ratedependent elastoplasticity. Computer Methods in applied mecchanis and engineering, 48:101-118.

Suiker, A. S. J. and Borst, R. D. (2003). A numerical model for the cyclic deterioration of railway tracks. International Journal for Numerical Methods in Engineering, 57:441 - 470 .

Wilkins, M. L. (1964). Calculation of elasto-viscoplastic flow. Academic Press, New York. 
List of Tables 


\section{List of Figures}

1 Deformable cylinder under triaxial loading

2 Variation of the deviatoric stress with respect to the axial strain in case of quasistatic triaxial loading of a cylindrical sample with unit radius and length

3 Variation of the hydrostatic pressure with respect to the axial strain

4 Variation of the quadratic stress with respect to the radial strain in case of quasistatic triaxial loading

$5 \quad$ Variation of the hydrostatic pressure with respect to the radial strain in case of quasistatic triaxial loading

$6 \quad$ Effect of the rate dependency on the response of a confined granular sample

$7 \quad$ Numerical accuracy of the model in case of typical cyclic triaxial testing

8 Cyclic radial displacement versus deviatoric stress 32

$9 \quad$ Cyclic axial displacement versus deviatoric stress 33

10 Variation of the volume with respect to time, logarithmic overall decaying

11 Loading and boundary conditions on the flexible sample

12 Response of a partially confined cylindrical sample to cyclic triaxial loading under different frequencies

13 Accumulated vertical permanent deformation along the surface of the sample for different frequencies 


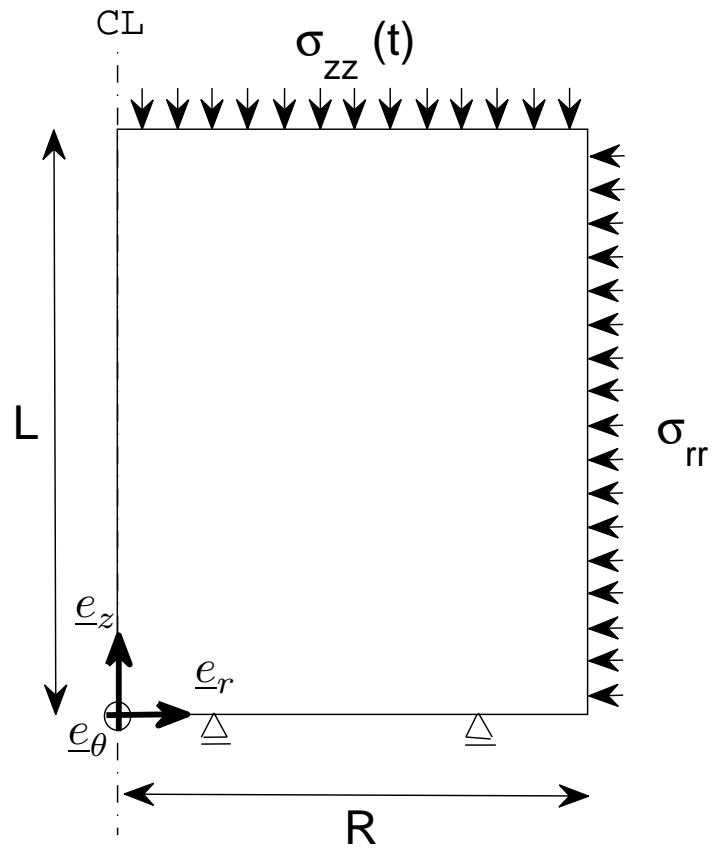

Fig. 1. Deformable cylinder under triaxial loading 


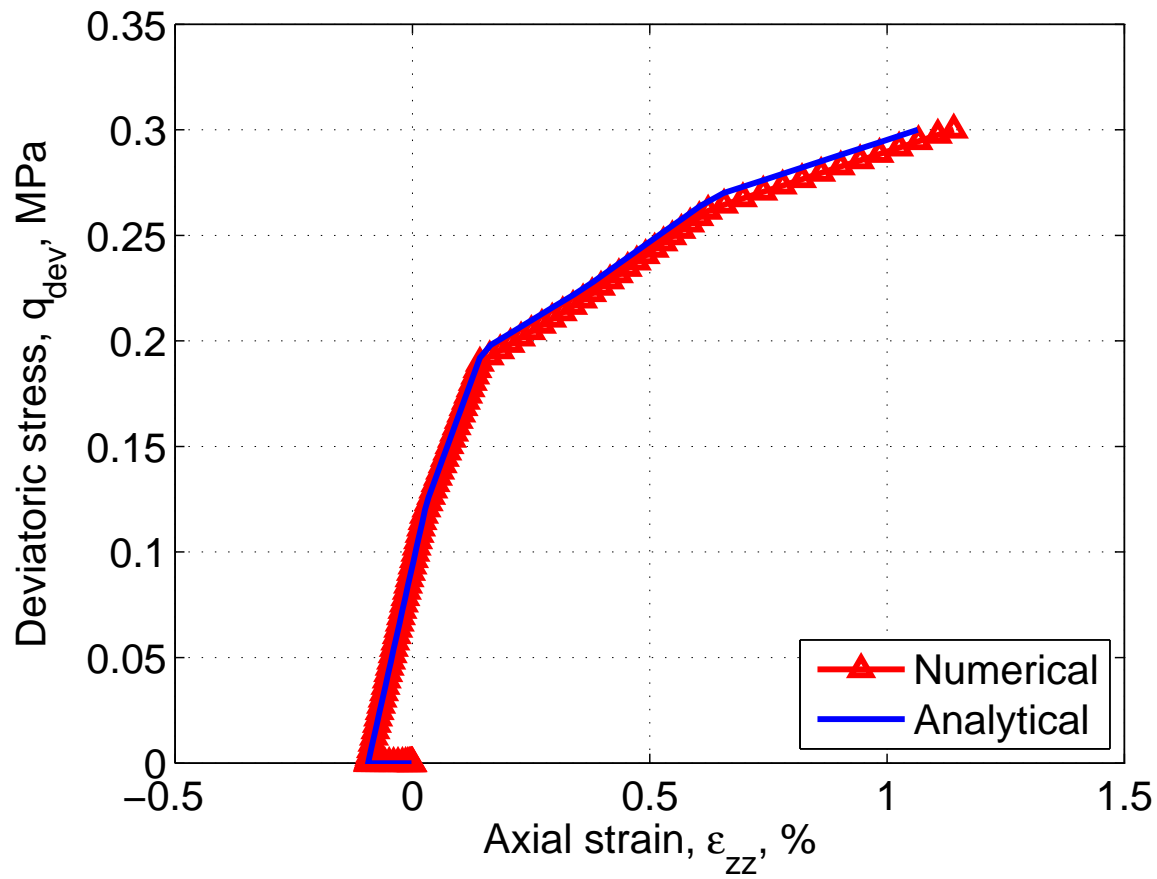

Fig. 2. Variation of the deviatoric stress with respect to the axial strain in case of quasistatic triaxial loading of a cylindrical sample with unit radius and length 


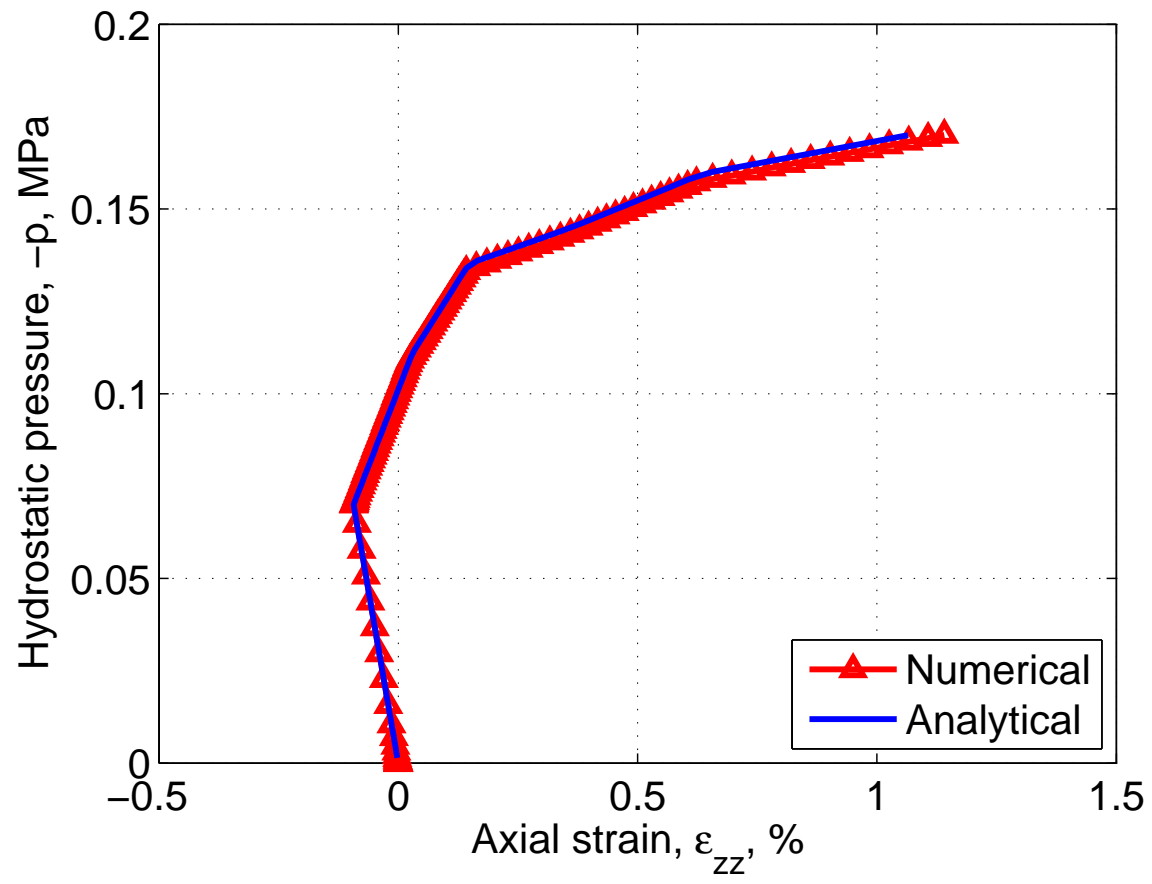

Fig. 3. Variation of the hydrostatic pressure with respect to the axial strain 


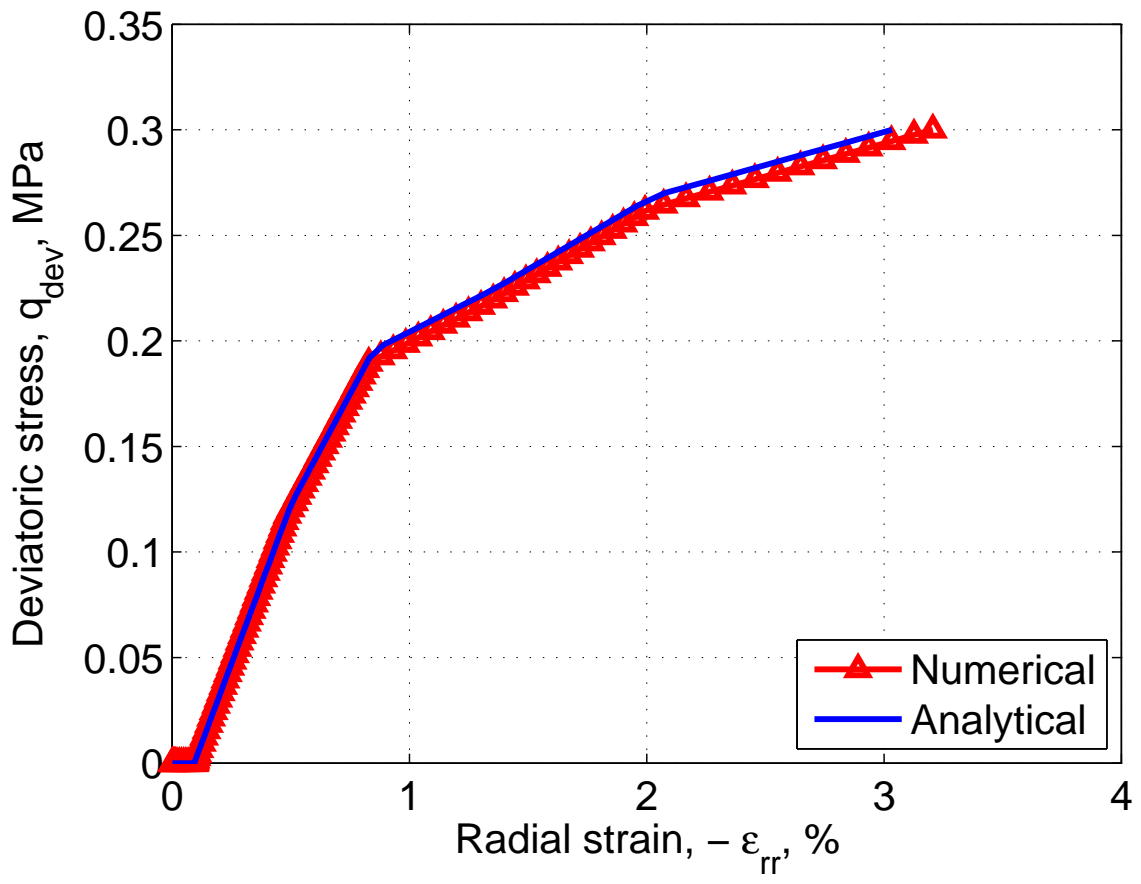

Fig. 4. Variation of the quadratic stress with respect to the radial strain in case of quasistatic triaxial loading 


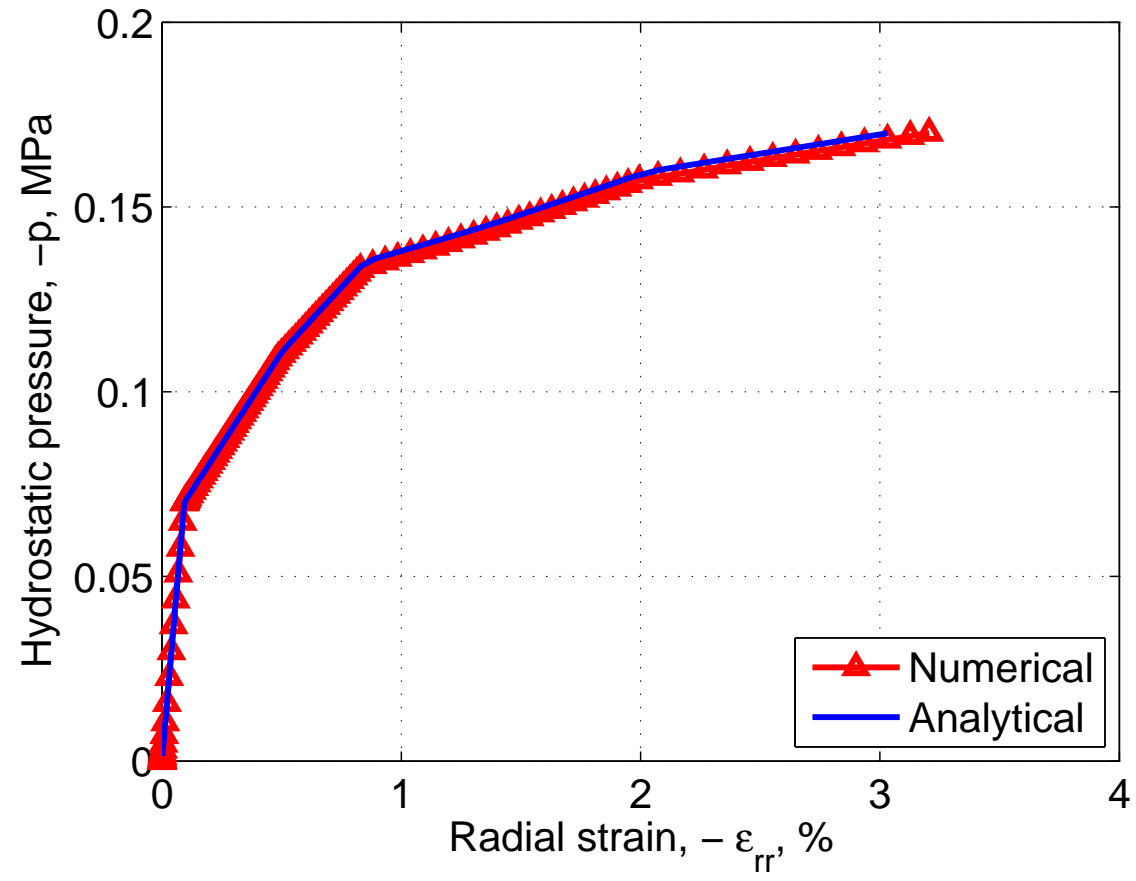

Fig. 5. Variation of the hydrostatic pressure with respect to the radial strain in case of quasistatic triaxial loading 


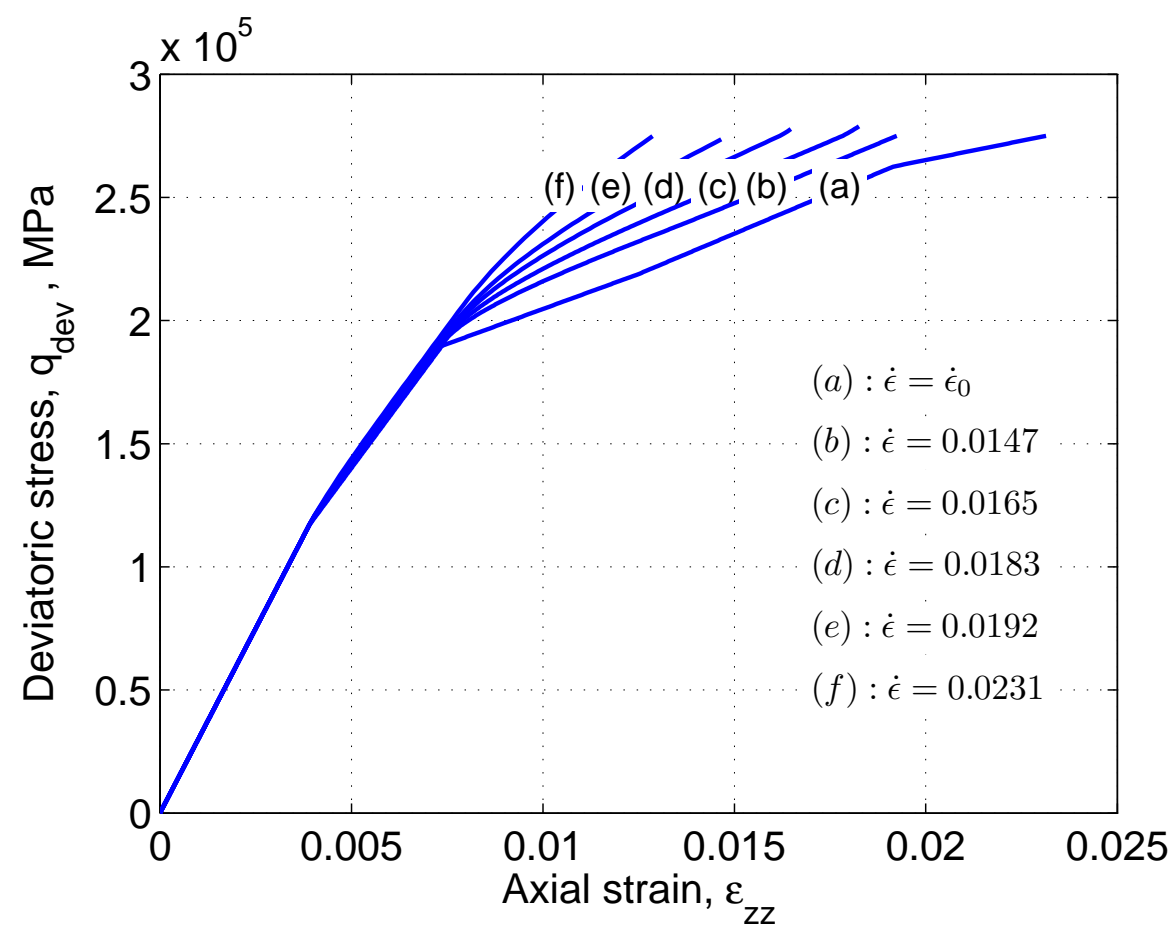

Fig. 6. Effect of the rate dependency on the response of a confined granular sample 


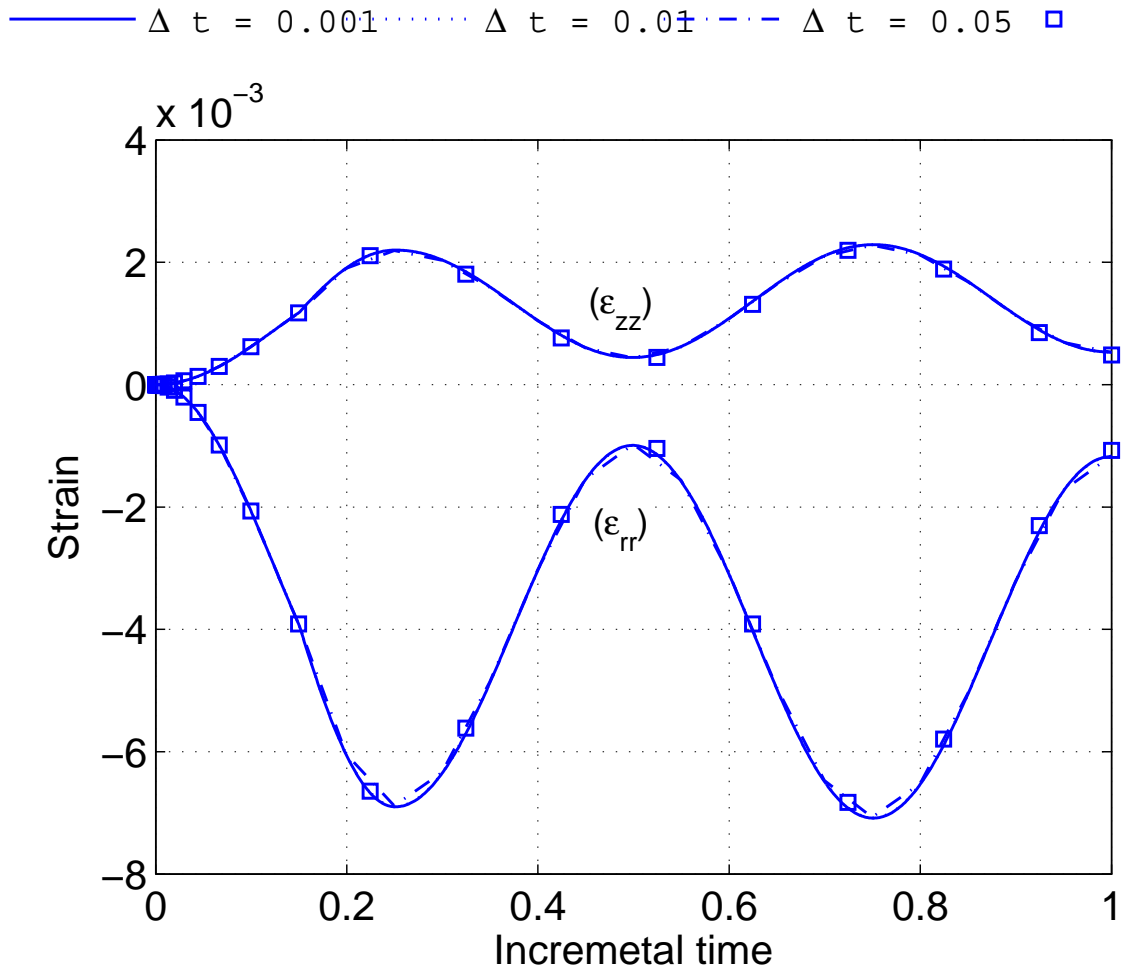

Fig. 7. Numerical accuracy of the model in case of typical cyclic triaxial testing 


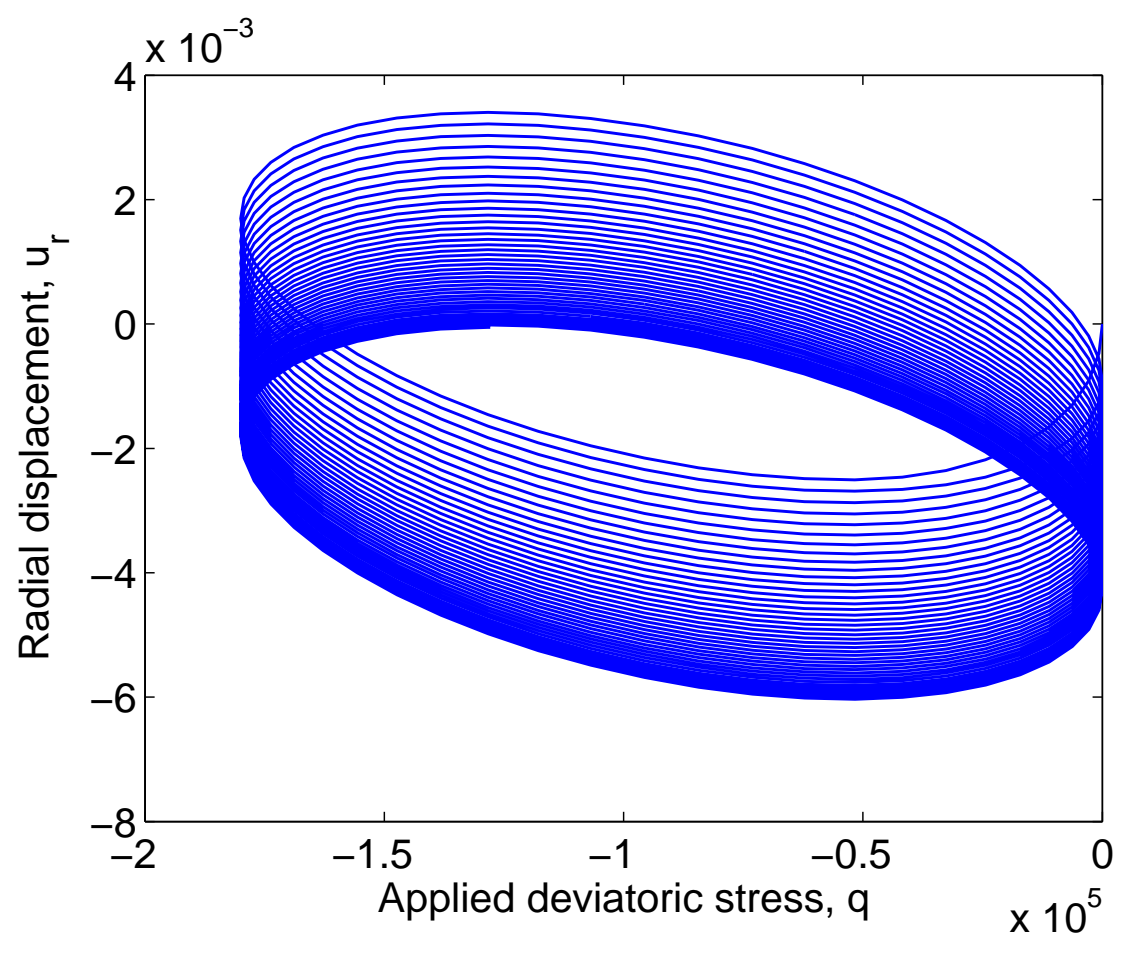

Fig. 8. Cyclic radial displacement versus deviatoric stress 


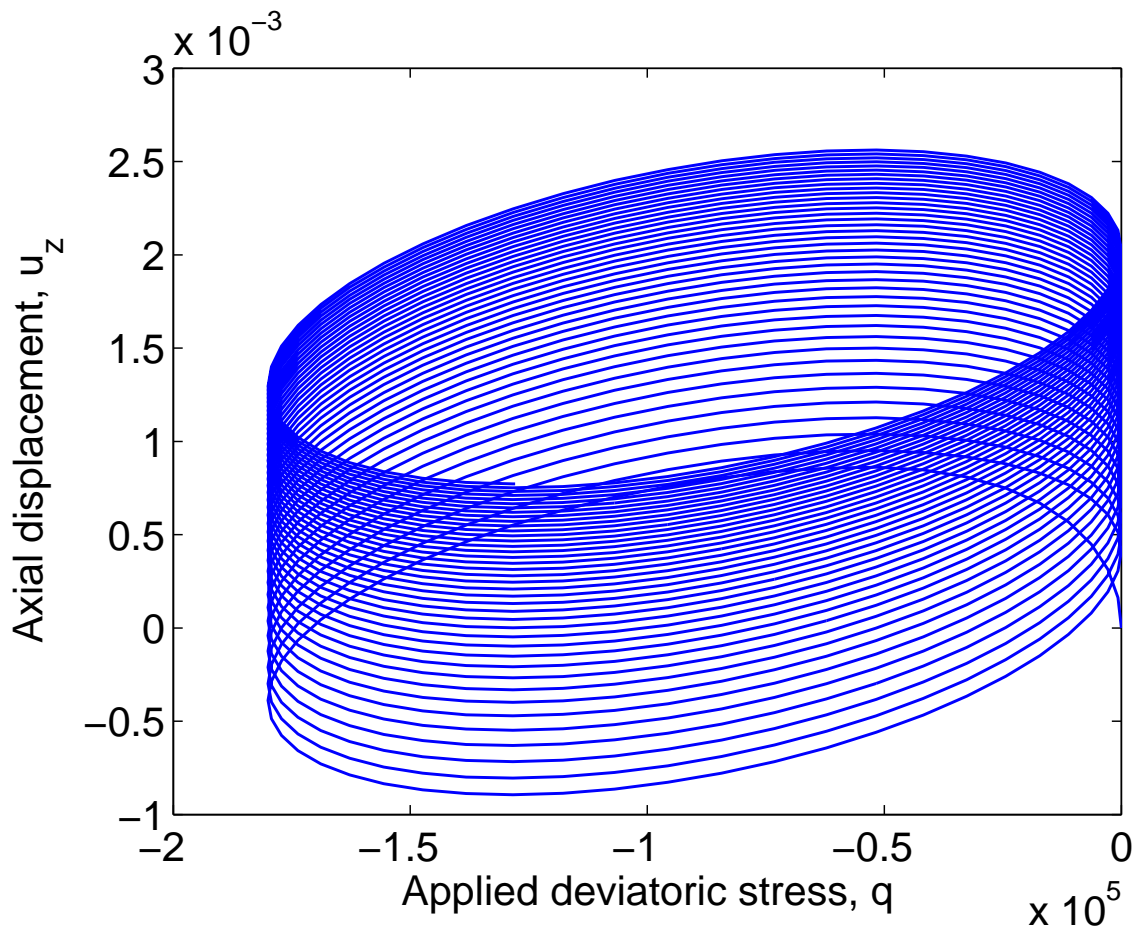

Fig. 9. Cyclic axial displacement versus deviatoric stress 


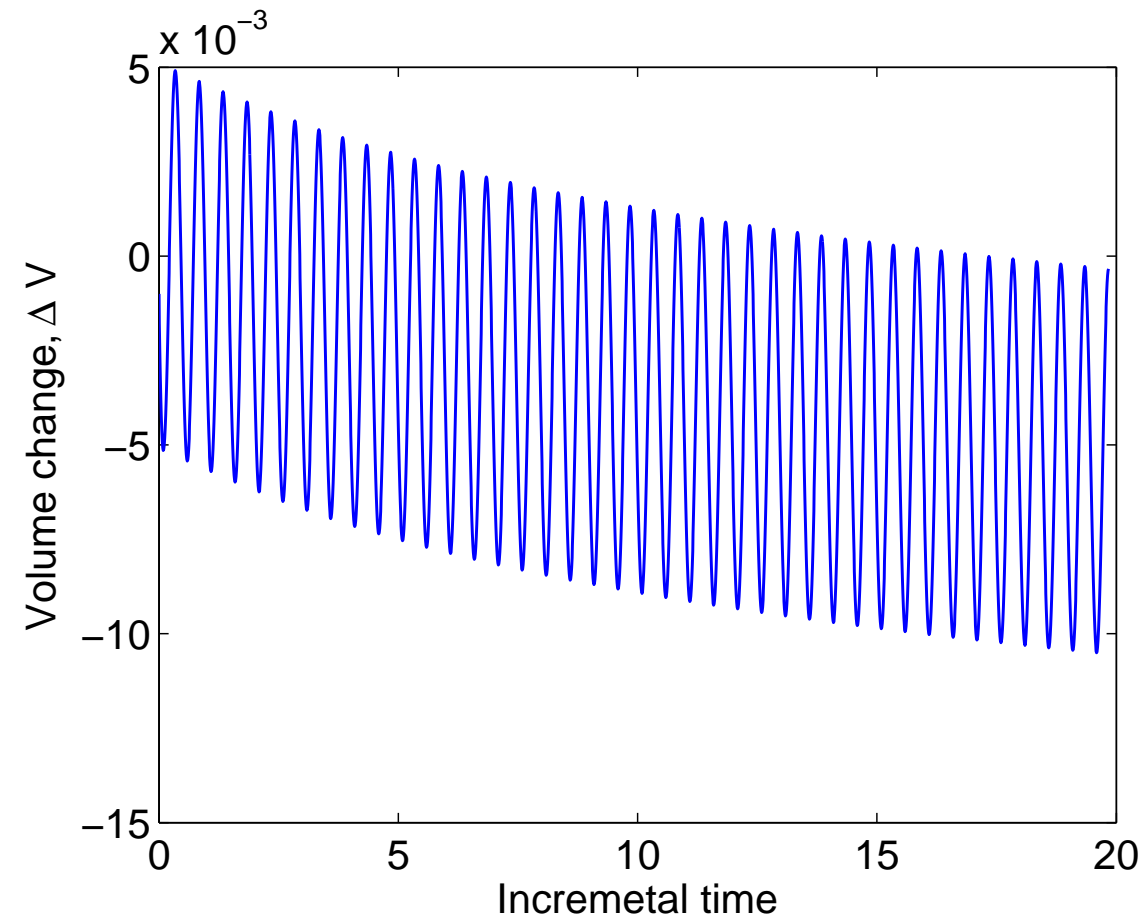

Fig. 10. Variation of the volume with respect to time, logarithmic overall decaying 


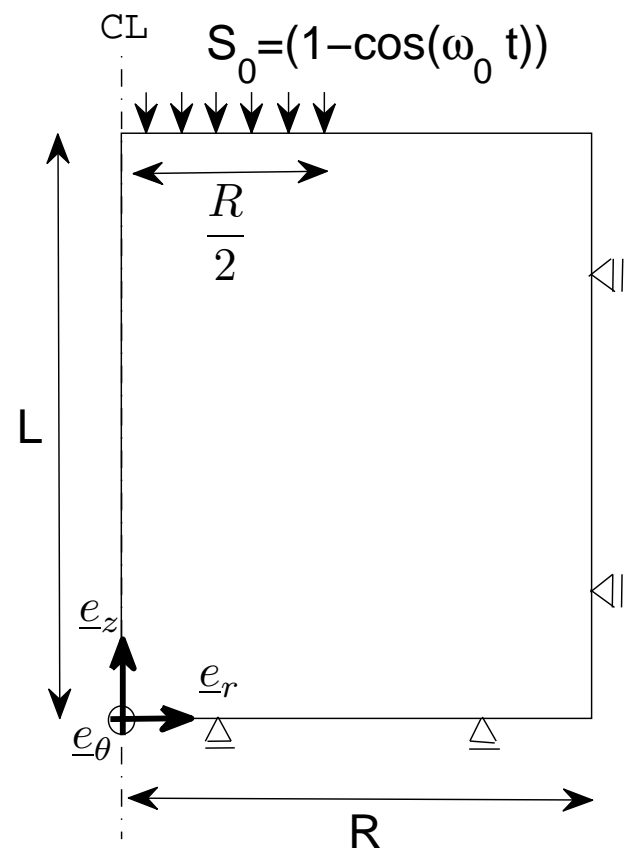

Fig. 11. Loading and boundary conditions on the flexible sample 


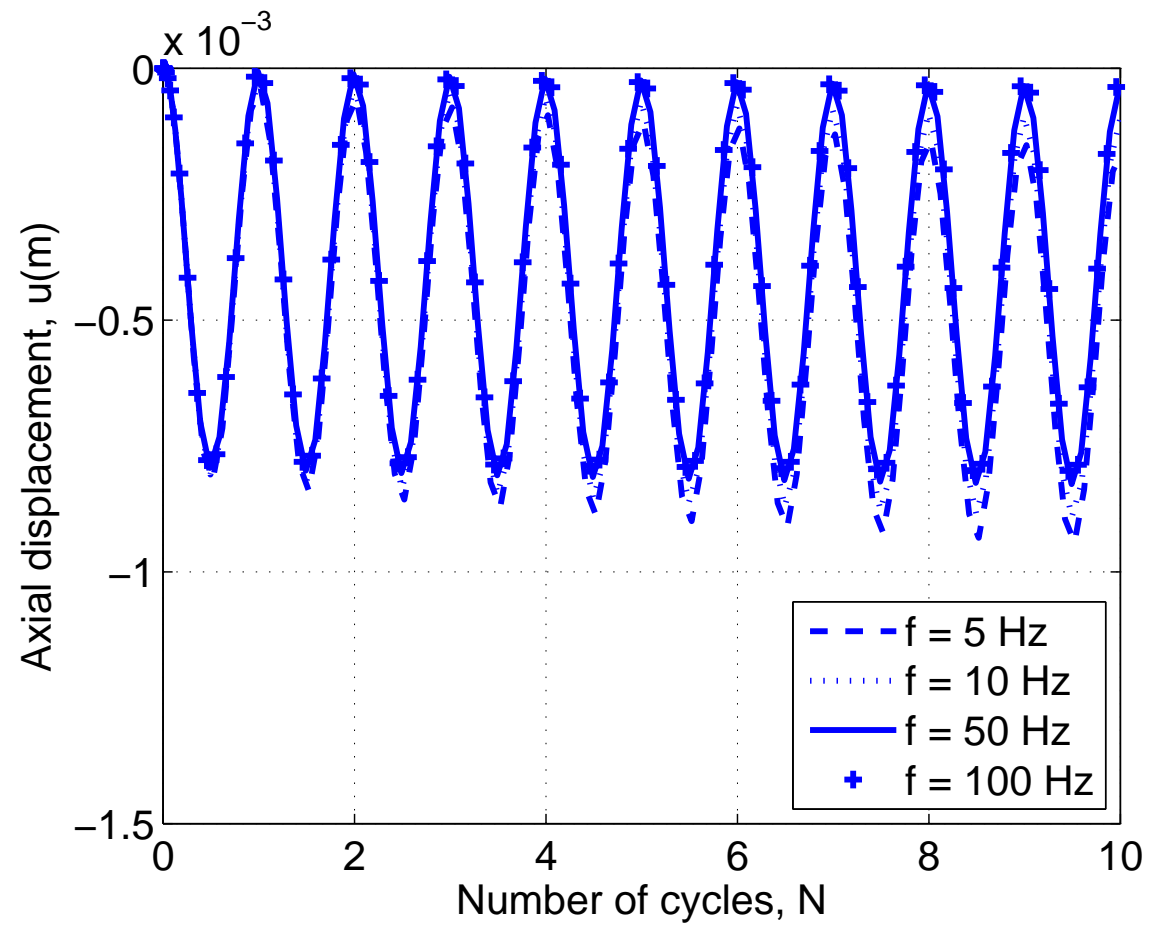

Fig. 12. Response of a partially confined cylindrical sample to cyclic triaxial loading under different frequencies 


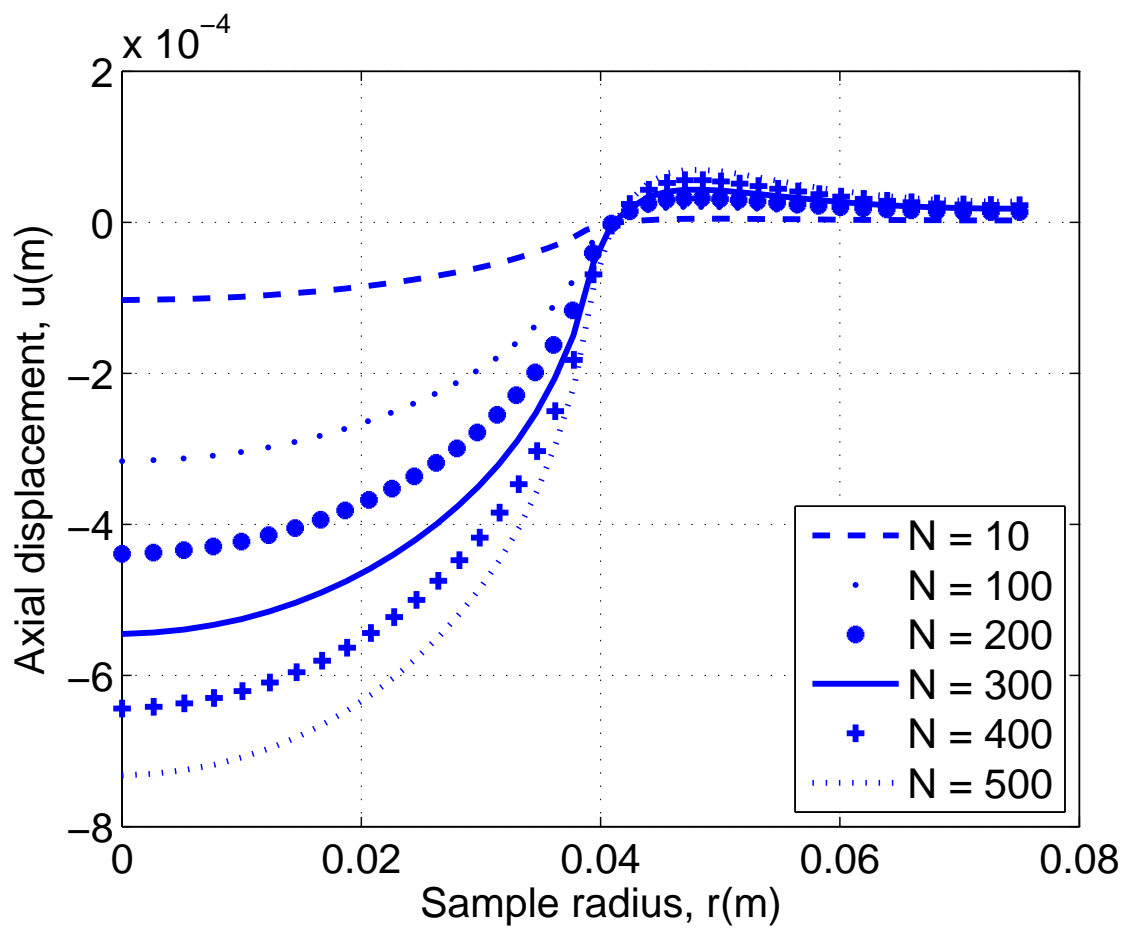

Fig. 13. Accumulated vertical permanent deformation along the surface of the sample for different frequencies 